$15^{\text {th }}$ International Conference on

AEROSPACE SCIENCES \& AVIATION TECHNOLOGY,

$\boldsymbol{A S A T}$ - 15 - May 28 - 30, 2013, Email: asat@mtc.edu.eg,

Military Technical College, Kobry Elkobbah, Cairo, Egypt,

Tel: +(202) 24025292-24036138, Fax: +(202) 22621908

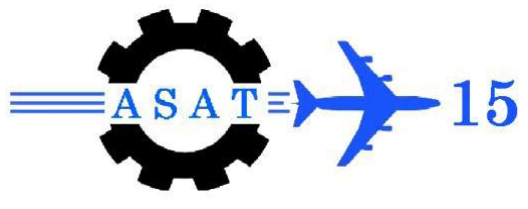

\title{
Surface Wave and Mutual Coupling Reduction between Four Element Linear MIMO Array Antenna
}

\author{
$\left\{\text { M. A. Abdalla }{ }^{*} \text { A. M. Mahrous, A. A. Mitkees, and A. M. Ali }\right\}^{\dagger}$
}

\begin{abstract}
In this paper an EBG structure is introduced for coupling reduction between elements in a four-element linear MIMO array of patch antennas operating at $5.8 \mathrm{GHz}$ to obtain proper independent performance for the elements of the array. The EBG structure was first employed between two elements linear MIMO array of patch antennas to assure functionality. Then, it was employed between the elements of the final four-element MIMO array antenna, simulated using a full wave simulation tool (HFSS). The proposed MIMO antenna is characterized by separation $0.5 \lambda_{0}$ between side by side elements. The array antenna performance was examined, in both cases, using electromagnetic full wave simulation. The final proposed four-element array antenna was simulated, using HFSS for coupling reduction validation. Results confirm that mutual coupling between each two side by side elements has been reduced by $4-13 \mathrm{~dB}$.
\end{abstract}

Keywords: Surface waves, mutual coupling, MMO array antenna.

\section{Introduction}

High gain antennas are needed for many communication and radar applications. A good candidate for this purpose is to use array of antennas. The major drawback that array antennas, suffer from is the mutual coupling between there elements. This drawback arises between the array elements due to the space wave which is common to all types of array elements, and surface waves, which in rectangular patch antennas are strongly excited in Eplane when the antenna is operated in the fundamental mode ( $\mathrm{TM}_{10}$ for rectangular patches) as this mode excites the first propagating mode of surface wave $\left(\mathrm{TM}_{0}\right)$ [1].

Several attempts have been conducted to reduce the mutual coupling effect between antenna elements in array antennas. An important attempt includes the use of electromagnetic bandgap (EBG) structures to reduce the surface wave [1]-[9]. Many shapes of EBG slots have been studied for single element microstrip antenna such as circles, dumb-bells and squares. It has been demonstrated that the EBG structure will lead to a reduction in the side-lobe levels and improvements in the front to back ratio and overall antenna efficiency for the radiation pattern. The capability of the EBG structure is its capability of avoiding the propagation of electromagnetic waves in a well-defined frequency range. [1].

In this paper, the aforementioned technique was employed for coupling reduction between a four elements linear array antenna used for space diversity MIMO applications. Mutual coupling reduction has been performed by employing an EBG structure. The EBG structure is employed to reduce the surface wave between the same elements of the linear array. The operating frequency was selected as $5.8 \mathrm{GHz}$ for several wireless applications. The antenna

maaabdalla@gmail.com

SEgyptian Armed Forces, Egypt. 
performance and mutual coupling investigation has been checked, in cases, two-element array, and four-element array, using electromagnetic full wave analysis.

\section{Design Procedure}

The design of the proposed EBG structure was first examined on a two element array antenna as shown in Fig. 1 where the proposed structure is a dumbbell like structure. As shown in the figure, the position of the EBG structure is between the two elements of the MIMO antenna. The detailed dimension is pointed out on the figure.

As mentioned before, the design objective of such EBG structure is aimed to prevent, in its band gab, surface waves produced by each element of the array from reaching one another, by this way the mutual coupling between side by side elements is reduced allowing for proper independent performance for the elements of the MIMO antenna.

Two cases were studied, for step by step approach to the final design. The first one was employing the proposed EBG structure in between the elements of a two-element MIMO array. For comparison, the mutual coupling between the two elements, while employing the EBG and while not employing the EBG structure, is investigated.

The single element of the conventional microstrip patch antennas was incorporated for the sake of antenna array antenna fabrication. The antenna was designed to operate at $5.8 \mathrm{GHz}$ which can be distinguished by reflection coefficient less than $-10 \mathrm{~dB}$ at that frequency. The simulated results for the two cases are plotted in Fig. 2. As shown in the figure, the mutual coupling, represented by the transmission coefficient from one port of single element to the other $\left(\mathrm{S}_{21}\right)$, is reduced from $-18 \mathrm{~dB}$, while not employing the EBG structure, to almost $-24 \mathrm{~dB}$ while employing the proposed EBG structure.

For further investigation, the field distribution throughout the substrate and the surface of the ground was simulated and depicted in Fig. 3. Fig. 3 (a) demonstrates the distribution of the electric field before employing the EBG structure, it can be seen that the field couples from one element to the other by the means of a surface wave that is bound to the surface of the ground. On the other hand, Fig. 3 (b) demonstrates the distribution of the electric field after employing the proposed EBG structure. It can be seen from the figure that the EBG structure acts as a barrier for the surface wave coupling from one element to the other and forms the main contribution for the mutual coupling between the elements.

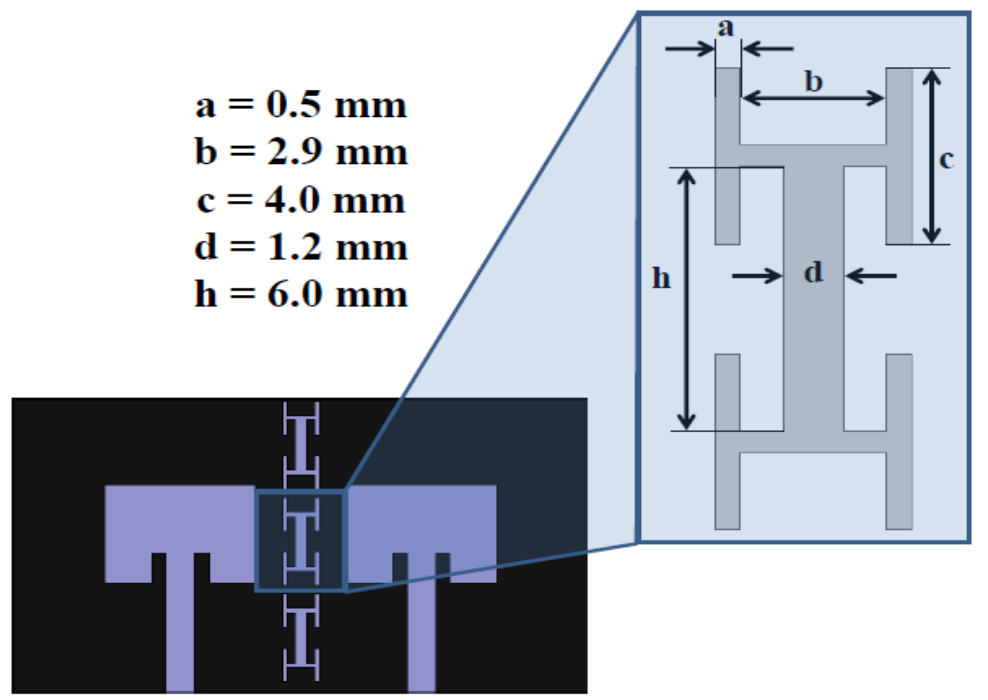

Fig. 1 The configuration of the EBG structure used between the two elements MIMO linear antenna. 


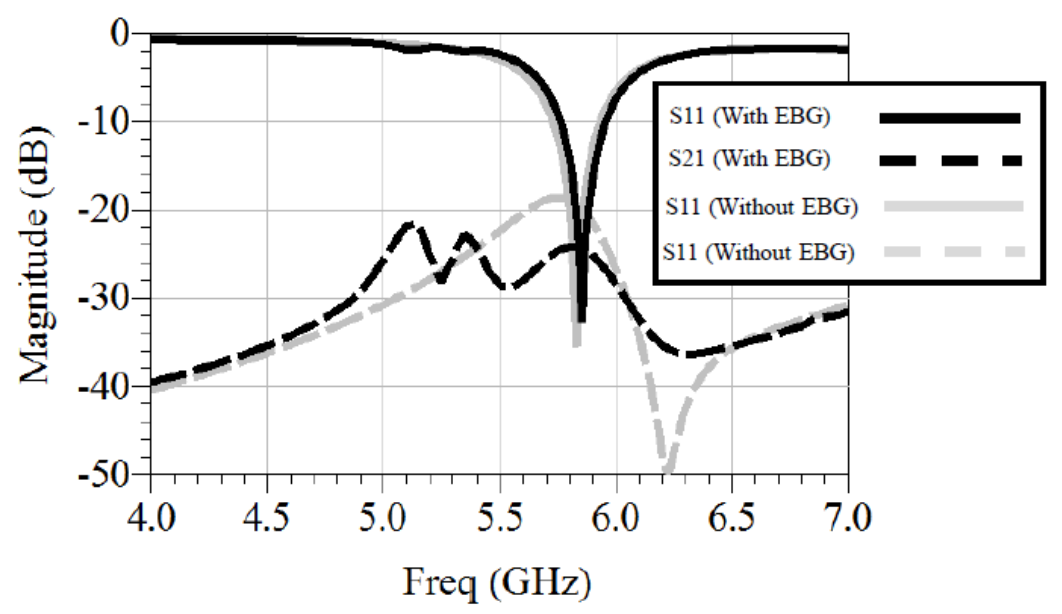

Fig. 2 The scattering parameters magnitudes of the two elements arrays MIMO antenna with and without using EBG.

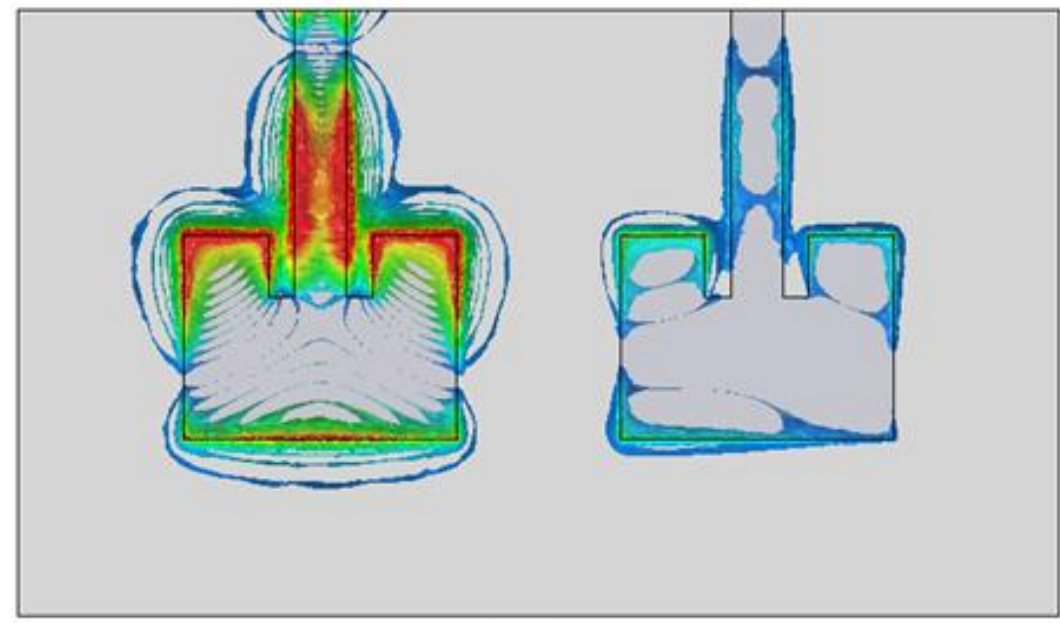

(a)

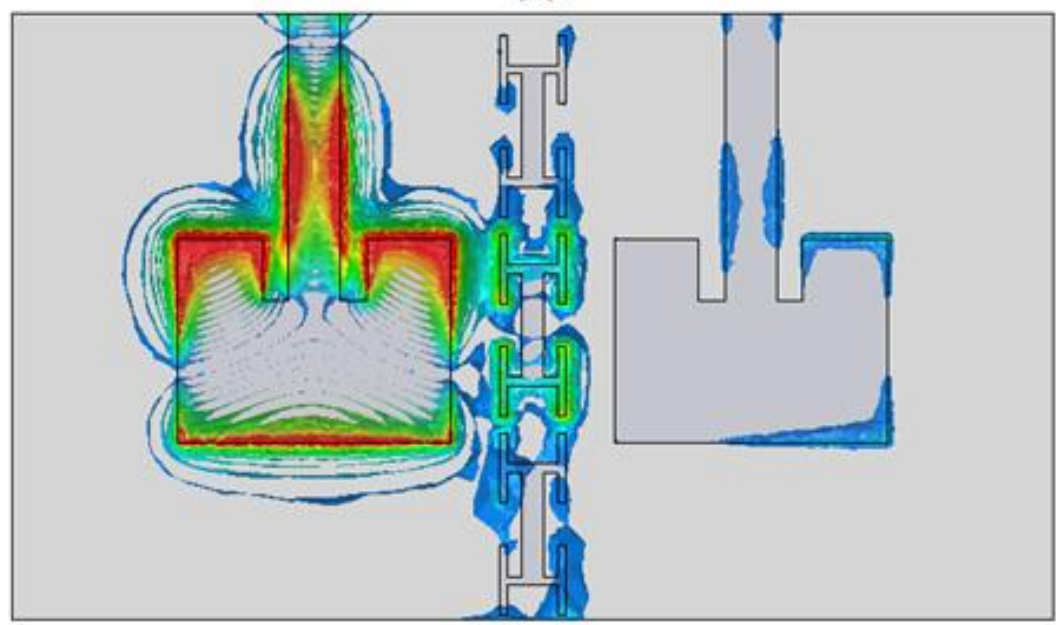

(b)

Fig. 3 The field distribution (a) before (b) and after applying the Proposed EBG structure. 


\section{The Results and Discussions}

The second case study was employing the proposed EBG structure to a four-element linear MIMO array of patch antenna. Fig. 4 demonstrates the structure of the four-element array incorporated with the proposed EBG structure in between the array elements. This time the mutual coupling between nearby elements is examined for the functionality of the EBG structure.

The reflection coefficient and the mutual coupling between each two side by side elements are depicted in Fig. 5 assuming the excitation is running from port 2 (P2) only. Fig.5 (a) demonstrates the reflection coefficient and mutual coupling between side by side elements before employing the proposed EBG structure in between the elements of the MIMO array antenna, and Fig. 5 (b) demonstrates them after employing the proposed EBG structure. It can be seen from the figure that the operating frequency of the antenna hasn't been affected after employing the EBG structure. Also, it can be seen that the mutual coupling between element number 1 and element number 2, configured by S21, has reduced about $4 \mathrm{~dB}$, at the specified operating frequency, after incorporating the EBG structure. The mutual coupling between element number 2 and element number 3, configured by S32 has reduced by about $6 \mathrm{~dB}$ at the specified operating frequency. Finally, the mutual coupling between element number 4 and element number 3 , configured by $\mathrm{S} 43$, has reduced by about $13 \mathrm{~dB}$ at the specified operating frequency. More investigation on the mutual coupling reduction has been carried out by demonstrating the field profile throughout the substrate and upon the surface of the ground as shown in Fig. 6. The figure demonstrates the two cases of not using and using EBG structure as shown in Fig. 6 (a), and (b) respectively. In both cases the field was excited at port 2 (P2) toward the other ports. As shown in Fig. 6 (a), it can be seen that the excited field exist significantly between the elements of the MIMO array antenna. On the other hand, in Fig. 6 (b), when employing the EBG structure, a significant phenomenon is clear. That is the field between the elements of the MIMO array antenna is concentrated around the EBG structure, which acts as a barrier, prevents it from being coupled the nearby antenna element.

\section{Conclusion}

An EBG structure was proposed to reduce the mutual coupling between the elements of a four-element MIMO array of patch antennas operating at $5.8 \mathrm{GHz}$. The proposed EBG structure was firstly employed in a two-element MIMO array of patch antennas operated at $5.8 \mathrm{GHz}$. It showed $5 \mathrm{~dB}$ reduction in the mutual coupling between the elements of the array configured by the transmission coefficient from port 1 (P1) to port 2 (P2). For more investigation the field distribution was depicted to ensure the functionality of the EBG structure, which is preventing the surface waves from being coupled from one element to the other. Secondly, the proposed EBG structure was incorporated upon a four-element linear array antenna. The mutual coupling between each side by side two elements was examined to show $4 \mathrm{~dB}, 6 \mathrm{~dB}$, and $13 \mathrm{~dB}$ reduction in mutual coupling between elements 2 and 1 (S21), 3 and 2 (S32), and 4 and 3 (S43), respectively. Again field profile throughout the substrate and upon the ground surface was depicted for further investigation on the functionality of the proposed EBG structure. This showed how the proposed EBG structure acts as a barrier to the surface wave preventing it from being coupled from one element to the other 


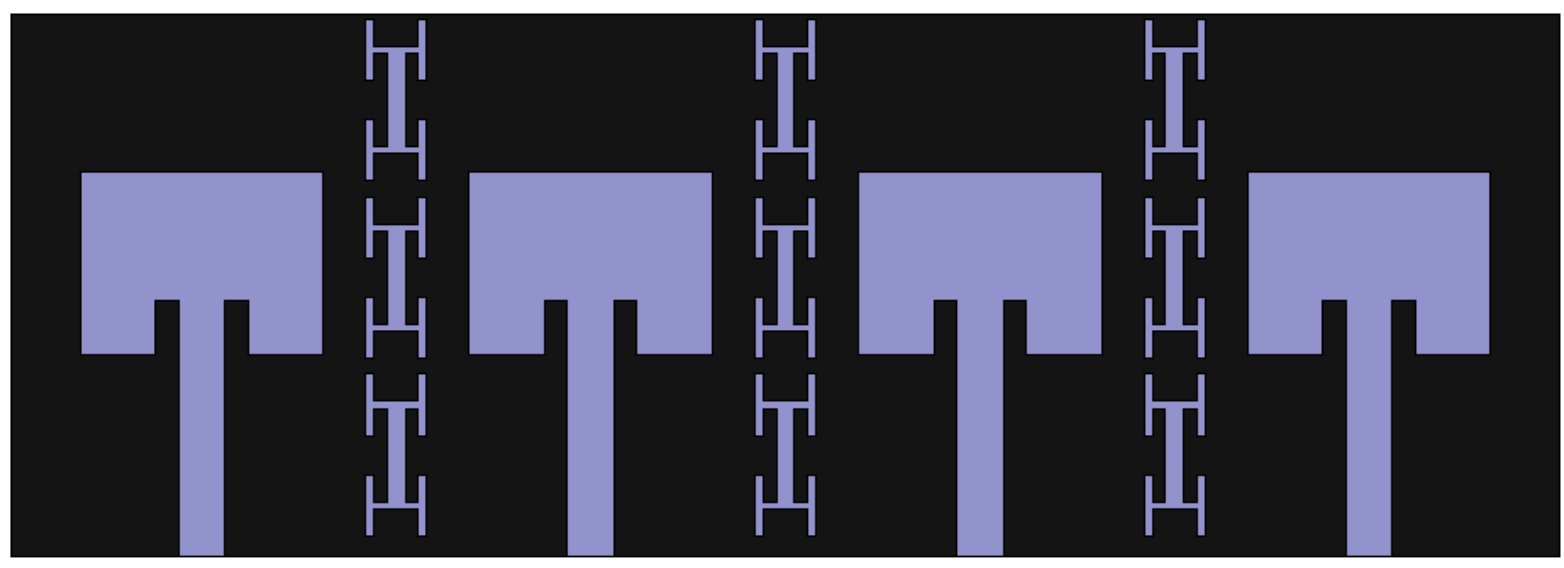

Fig. 4 Structure of the four-element array incorporated by the EBG structure.

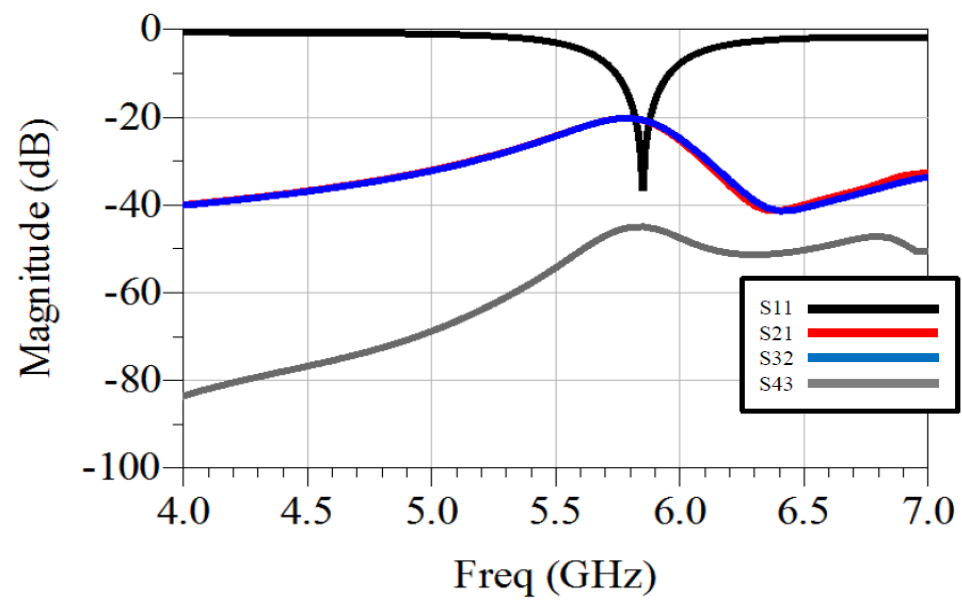

(a)

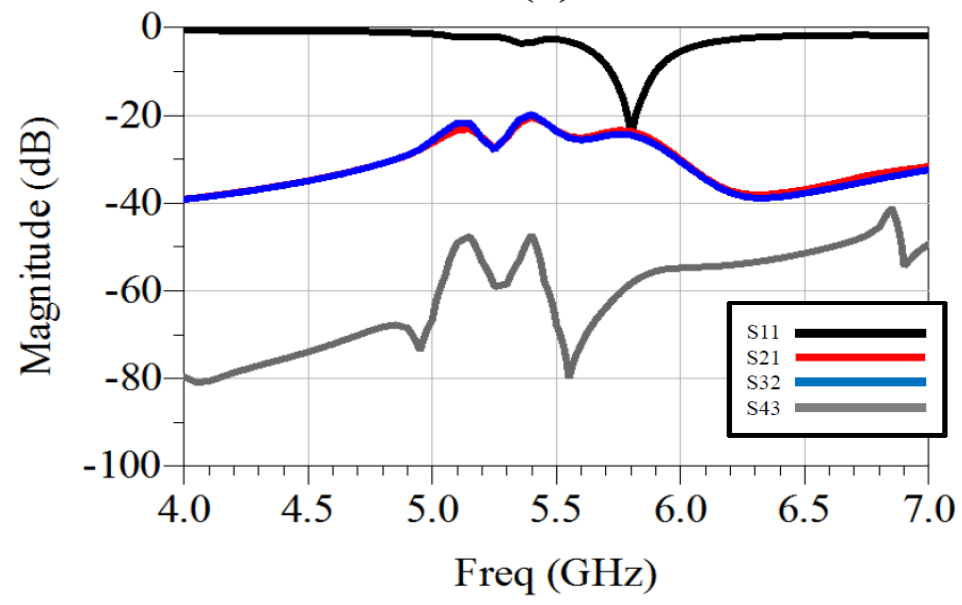

(b)

Fig. 5 Reflection coefficient and mutual coupling (a) before and (b) after applying the EBG structure. 


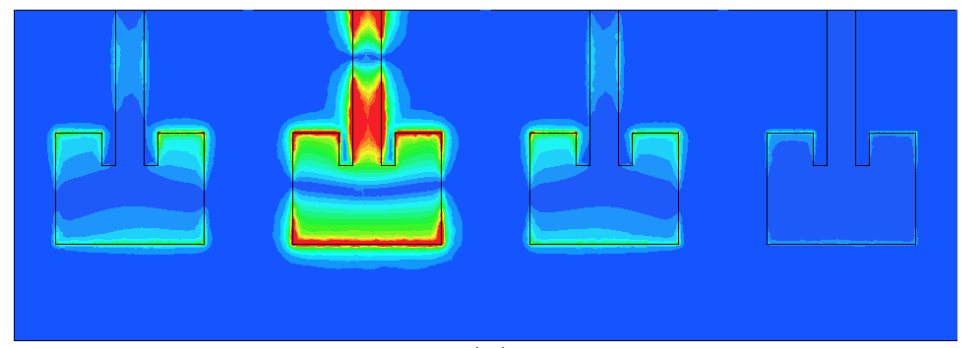

(a)

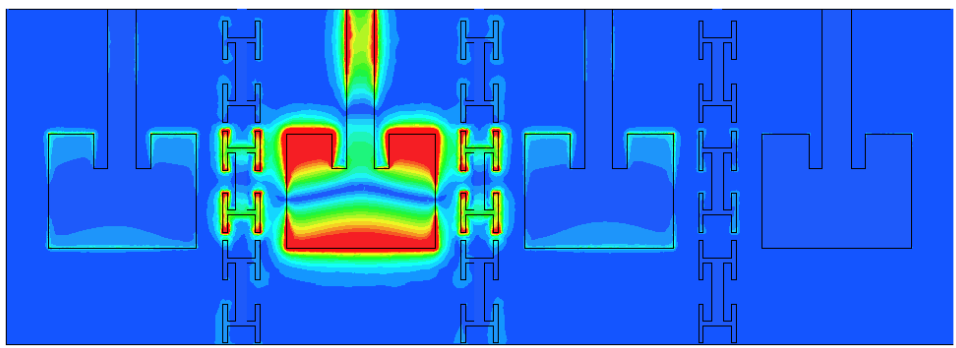

(b)

Fig. 6 The field distribution for the final proposed MIMO antenna

(a) before (b) and after applying the Proposed EBG structure.

\section{References}

[1] Dan Sievenpiper, "High-Impedance Electromagnetic Surfaces with a Forbidden Frequency Band", IEEE Transaction on MTT, VOL. 47, NO. 11, November 1999.

[2] $\mathrm{Yu}, \mathrm{A}$. and $\mathrm{X}$. Zhang, "A novel method to improve the performance of microstrip antenna arrays using dumbbell EBG structure," IEEE Antennas and Wireless Propagat. Lett., Vol. 2, 170-172, 2003.

[3] Horii, Y. and M. Tsutsumi, "Harmonic control by photonic bandgap on microstrip patch antenna," IEEE Microwave Guided Wave Lett., Vol. 9, 13\{15, 1999.

[4] Yang, F. and Y. Rahmate-samii, Mutual coupling reduction of microstrip antennas using electromagnetic bandgap structure," IEEE Antenna and Propag. Soc Int. Symp., Vol. 2, 478-481, Boston, MA, 2001.

[5] Yu, A. and X. X. Zhang, \A novel 2D electromagnetic band-gap structure and its application in microstrip antenna arrays," Proc. 3rd Int. Conf. Microwave and Millimeter Wave Tech. ICMMT, 580-583, 2002.

[6] Yang, L., Z. Feng, F. Chen, and M. Fan, "A novel compact EBG structure and its application in microstrip antenna arrays," IEEE MTTS Digest, 1635\{1638, 2004.

[7] Yang, F. and Y. Rahmate-Samii, \Applications of electromagnetic band gap (EBG) structures in microwave antenna designs," Proc. 3rd Int. Conf. Microwave and Millimeter Wave Tech., 528-531, 2002.

[8] Salehi, M., A. Motevasselian, A. Tavakoli, and T. Heidari, Mutual coupling reduction of microstrip antennas using defected ground structure," 10th IEEE International Conference on Communication Systems (ICCS), Oct. 1-5, 2006.

[9] Eva Rajo-Igalesias, "Design of a planar EBG Structure to reduce Mutual Coupling In Multilayer Patch Antennas", Loughborough Antennas And Propagation C, Loughborough, UK, 2007. 